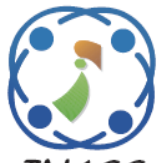

\title{
Image Compression Using Swarm Intelligence
}

\author{
Shahbaa I. Khaleel ${ }^{1 *}$ \\ ${ }^{1}$ Software Department, College of Computer Science and Mathematics, Mosul University, Iraq \\ * Corresponding author's Email: shahbaaibrkh@uomosul.edu.iq
}

\begin{abstract}
As a result of the development in multimedia technology and direct dealing with it in social media, it has led to interest in the techniques of compacting color images because of their importance at present. Since image compression enables the representation of color image data with the fewest number of bits, which reduces transmission time in the network and increases transmission speed. To ensure the compression process is performed without loss of data, the lossless compression methods are used because no data is lost during the compression process. In this research, a new system was presented to compress the color images with efficiency and high quality. Where the swarm intelligent methods were used, as well as hybridizing it with fuzzy using the Gustafson kessel fuzzy method to improve the clustering process and create new clustering methods with fuzzy swarm intelligence to obtain the best results. Swarm algorithms were used to perform the process of clustering the image data to be compressed and then obtaining a clustered data for this image data. In contrast, a lossless compression method was used to perform the encoding of this clustered data where the huffman method was used for encoding. Four methods were applied in this research to different color and lighting images. The PSO swarm intelligent was used, which in turn was hybridized with the Gustafson kessel fuzzy method to produce a new method for fuzzy particle swarm (FPSO), as well as the grey wolf optimization method GWO, which was hybridized with Gustafson kessel and obtained a new method, which is the fuzzy grey wolf optimizer FGWO, and the results were graded efficiently from the first to the fourth method, where the FGWO method with the huffman was the most efficient depending on the standards measurement that were calculated for all methods, the compression ratio was high in this new method, in addition to the standards of MSE, RMSE, PSNR, etc. among the important measurements of the compressing process.
\end{abstract}

Keywords: Image compression, Swarm intelligence, Particle swarm optimization PSO, Grey wolf optimization GWO, Fuzzy logic, Huffman encoding.

\section{Introduction}

Due to the technological development and the use of the Internet to send and deal with images, it necessitated facilitating the task in sending them. The process of compressing images is the best example of these facilities, as the best transmission of the image should not take much time, so it was necessary to compress the color image in order to take a few bytes in its representation in addition to reducing the time required to send it $[1,2]$. The compressing process is converted the images into smaller files to ensure efficient storage and transmission. As one of the advanced technologies of multimedia technology where the use of multimedia is common in all social media and on a large scale. Where the high speed of transport has acquired great importance at the present time, which led to interest in the pressing operations and its development to obtain the most efficient way [2-4]. The main performance measure for image compression is the compression ratio. And the process of compressing images is with two types of pressing with loss and pressing without loss, and the second type is the best because a file identical to its original is obtained with high accuracy [1,5]. Swarm Intelligence It is an intelligent technique centered around the study of the collective behavior of decentralized systems, where this technique simulates social behavior in a swarm in order to solve complex problems. Where the swarm solves complex problem through a number of its members without central control. In general, all techniques of swarm 
intelligence depend mainly on the population, where there is a set of possible solutions, so the optimal solution is sought. population members within the overall search space are changing their locations. There are several intelligent swarm algorithms $[6,7]$. Image clustering is one of the basic steps in image analysis and image processing applications. The goal of this process is to obtain meaningful areas and this is important before any treatment that occurs at the higher level of the image. Clustering is a method of grouping similar data into different groups or clusters, each group must be similar, and different groups must be non-containers of shared data. The choice of criteria for homogeneity or similarity plays an important role in the accuracy of the cluster result [7, 8]. In this research, a number of topics were linked, compression, swarm intelligence, and data clustering. Where the process of pressing for color images was performed with high efficiency through the use of swarm intelligence techniques in conducting the clustering process for color images data and then using the Hufman coding to complete the pressing process. The swarm intelligent algorithms used in this research to conduct the clustering are the particle swarm algorithm and the grey wolf algorithm, and these two algorithms were developed by hybridizing them with fuzzy logic by making use of the fuzzy membership function to make the clustering process by swarm intelligent flexible and high accuracy. Where a process of reduced the color image data was carried out using a clustering process that shrinks the data and reduces the duplicates in it, and in order to ensure that the data is not lost during the reduction, the best algorithms are used for this. Where the intelligence of the swarm was used because of its importance in finding the best solution to all problems, and that the clustering image in these methods despite the reduction of its data, but its high quality and conformity to the original image is excellent, due to the efficiency of the methods of swarm intelligence in the clustering process where the principle of the traditional clustering algorithm was used to find the Euclidean distance between each pixel with the centers of the clusters and the inclusion of this calculation within the equation for calculating the fitness function of the particle swarm algorithm and the grey wolf optimization algorithm, which produced excellent clustered data, and then this data was coded using the huffman algorithm, thus obtaining high-quality compressed images. Also in this research, fuzzy logic features were used to improve the work of swarm intelligence algorithms by using membership function values that help the pixel go to the class to which it belongs faster, in addition to using mahalanobis distance in finding the similarity value between pixels and the centers of clusters to ensure the data cluster correctly and quickly. The membership function has a primary advantage in improving the clustering process, because each pixel has its own class belonging value, which can be used to classify the pixel correctly. Here, the particle swarm algorithm was hybridized with the Gustafson kessel algorithm and a new method called FPSO was obtained and its work was better. The fuzzy Gustafson kessel algorithm was also hybridized with the grey wolf optimization algorithm and a new FGWO algorithm was produced, which proved its success in the clustering process and obtained the best results as shown in the tables and results and for various images of different dimensions and colors. Where the best value of the PSNR measure of the new FGWO hybrid method was obtained, as well as obtaining the best values for all measurements of this method. By using swarm intelligence with Gustafson Kessel fuzzy algorithm, it gave the research importance and efficiency in image compression compared to other research.

Section 2 includes the previous work, and the proposed system of this research is explained in section 3, and in section 4 the particle swarm algorithm is explained in detail and how it was employed to perform the clustering process, in addition to improving this method by hybridizing it with the fuzzy Gustafson kessel method and obtaining the proposed FPSO algorithm for the fuzzy clustering explained in section 5. Section 6 dealt with the grey wolf optimization algorithm that was used to perform a clustering operation on image data using Euclidean distance as a function of its fitness. In section 7 the proposed fuzzy hybrid method FGWO is presented in which mahalanobis distance was used to calculate the pixel similarity amount to the cluster's centers in addition to using the membership function to improve and accelerate the clustering process. Section 8 explains the coding process represented by Huffman's algorithm. Measurements for the compression process are described in section 9. Section 10 deals with all practical results that illustrate the robustness of the methods proposed in this research, and finally the conclusions are clarified in Section 11.

\section{Previous works}

Prabhjeet kaur and Er. Parminder Singh proposed a new compression technique that hybridized Particle Swarm Optimization (PSO) and the genetic algorithm (GA) for image compression based on wavelet transformation. Particle swarm performance depends mainly on elementary particles. When 
poorly selected particles that lead to bad results. The genetic algorithm does not guarantee global results (optimal solutions), but it is rich and powerful due to the presence of its mutations and crossover operators. These reasons led researchers to hybridized the particle swarm algorithm with the genetic algorithm [9], The proposed method was applied here on color and gray level images, and the values of the PSNR measure ranged between 32.6901 - 39.0999, while in our research and when applying the proposed methods (FGWO), the values of the PSNR measure ranged between 60.496-70.052 and thus quality of new methods was proven. Researchers S. keerthika, S. Vidhya have proposed a new method for image compression, which is a new advanced particle swarm using the fractal image compression method FIC that based on sorting. This is to speed up the encoding process and get high-quality recovered images. The image coefficients are extracted using DCT to classify the image. Then according to the coefficients of the domain areas, the search strategy for each domain block is determined using the proposed algorithm [10], When implementing this proposed method on the images, the highest PSNR values were obtained, which were 56.804, and the compression ratio was 44.342. Whereas in our research, better values of these measures were obtained where the values of the PSNR measure ranged from 60.496-70.052 and the values of the compression ratio ranged between 54.85-62.03, and thus the research methods proved their quality. Hongyi Li et al. Presented an intelligent method to feature extraction and clustering using a PSO swarm clustering algorithm and was applied to electromagnetic interference signal data. And then this method was compared with the traditional clustering methods such as K-mean, FCM and SOM, and it became clear from the results that the proposed method by researchers was superior to the algorithms that were compared with it, as well as the runtime was less [11]. Ibrahim Aljarah and others presented a developed algorithm based on grey wolf to perform data collection in various domains. The grey wolf algorithm was hybridized with a tabu search (TS), obtaining a robust clustering algorithm. It was tested on thirteen data sets, along with a statistical rank test to assess the efficiency of GWOTS compared to previous algorithms. From the results that were reached, it was found that the proposed method outperformed the previous methods [12]. The researchers Divya and Prakasha used multiple methods to perform a clustering process on medical images to discover diseases, as fuzzy cmeans, kmeans, spatial fuzzy means clustering, gustafson kessel and finally particle swarm optimization incorporative cmeans were used. These methods were the basis for diagnosing and discovering a brain tumor in humans, by relying on the clustered data of previous methods because of the importance of the clustering in many image processing methods and is considered the main stage in the application. In this research, the accuracy of the algorithms was tested by applying them to brain images, and the resolution was high [13]. Jamil As-ad et al. Presented an efficient method of data compression, as dependend on the method of data compression without loss, represented by huffman's algorithm, is considered the most common method of compressing data. The proposed method aims to reduce the size of the huffman coding tree and also to propose a new technique for efficient memory storage for huffman tree storage. From the results they reached, they proved that their proposed method is much better than previous and current methods of compressing data [1]. Researchers Chiranjeev Singh and Apratim Gupta proposed a new method of data compression and is considered an improvement to huffman algorithm, and the work of this algorithm is similar to Huffman algorithm where a tree is used to generate the codeword for each symbol and depends on the frequency of the symbol's presence. And that each codeword is alone, meaning it does not resemble the other codeword, which prevents ambiguity. A marker is used whose code word is unique and not ambiguous. The marker was used to encode the English capital letters using lowercase letters and vice versa [3].

\section{The proposed system}

In this research, a system has been proposed to perform the compressing process of color images with the lowest cost and the least time in addition to obtaining the best results for the compressed images in terms of their conformity with the original image in addition to obtaining the least storage space required to store the compressed data. Where a process was done to reduction the data of color images using the clustering process, which in turn reduction the data and reduces redundancy and prepares it to perform the pressing process. In order to ensure that the data is not lost while reducing it, the best algorithms have been used for that. Where the intelligence of the swarm was used because of its importance in finding the best solution for all problems. Here the particle swarm algorithm was used to perform the clustering, and this algorithm was hybridized with fuzzy logic to obtain an easy, flexible and precise clustering operation at the same time. Grey wolf algorithm was used to perform the clustering process for data and also was hybridized 


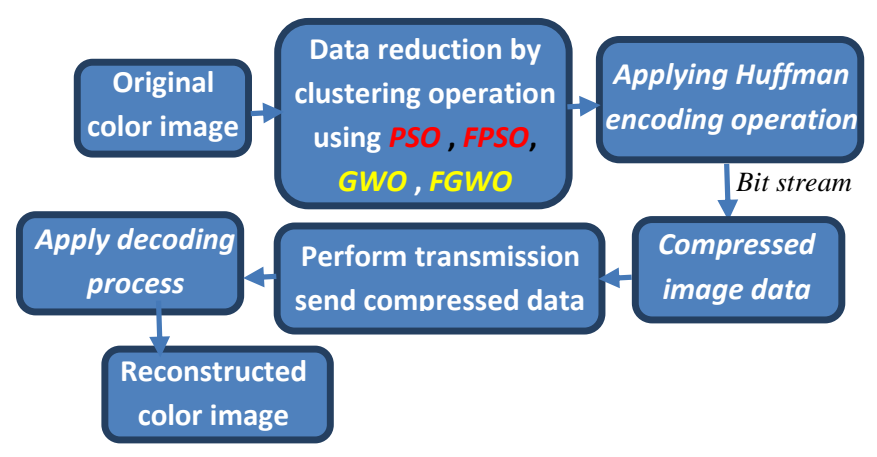

Figure. 1 Basic steps of the proposed system

with fuzzy logic to obtain a better algorithm than all previous methods. And then coding the clustered data using the lossless algorithm, which is the huffman that gave great results by pressing. The following diagram (Fig. 1) shows the basic steps of the proposed system.

Compression of color image data is one of the most important technologies at the present time for development of information technology. There are many methods used to compress data and make it more easy and portable. Here, in this research, two new methods are proposed that help in obtaining high-quality compressed images. Where the particle swarm algorithm has been developed by hybridizing it with fuzzy logic to form a new method for the clustering represented by FPSO, as well as proposing another hybrid method, FGWO, which has proven its worth and strength in the compression process, where, when applied to color images, the clustered images were obtained excellently and then coded and obtained data high quality pressed. In addition to using the two aforementioned methods without hybridization, their results were compared, and it was found that the last hybrid method was better than all methods, depending on the measures calculated for all the various images used in the research. In the following sections, all these methods will be explained.

\section{Particle swarm algorithm PSO}

The particle swarm algorithm is an algorithm that relies on its population, simulating the natural behavior of bird flocking in a computer program. Individuals are initially created with random solutions, called particles. Each of these particles has its own velocity [10]. The particles fly in the search space, where their velocity is continuously adjusted according to the behavior of the particles, so the particles have a tendency to fly towards the best solution in the search space. Each particle in the swarm has the following characteristics: [13], post $i$ : represents current location of particle. $v e_{i}$ : current velocity of particle. best_ini : best position taken by particle [14]. All particles have fitness values, which aim to improve the fitness function to find the best solution in the search space [15], where each particle uses its individual memory and the information it gained from the swarm as a whole to discover the best solutions. Where each particle adjusts its position according to its own practice and also according to its knowledge of its closest particle, as it benefits from the best location it and its neighbor encounter. Each particle's information is updated based on two values called best_g and best_in [16].

$$
\begin{gathered}
v_{i}^{i t e r+1}=\omega \times v e_{i}^{i t e r}+\gamma_{1} r v_{1}(\text { best_g } \\
\text { post } \left._{i}^{i t e r}\right)+\gamma_{2} r v_{2}\left(\text { best_in }_{i}-\text { post }_{i}^{i t e r}\right) \\
\text { post }_{i}^{i t e r+1}=\text { post }_{i}^{i t e r}+v e_{i}^{i t e r+1}
\end{gathered}
$$

Where ve represent velocity, $\omega$ represent inertia weight; $\gamma_{1}, \gamma_{2}$ are learning parameters (acceleration parameters); $r v_{1}$ and $r v_{2}$ represent random values (01); best_g position of particle that have best global fitness (best solution); best_in individual best solution.

In the algorithm of the swarm of particles that combined with traditional clustering process, each particle contains $\mathrm{k}$ from the cluster centers, so the swarm represents a group of solutions for the clustering process and the fitness function that reduces the error by reducing the distance between the clustering centers and the data that belong to them and enlarging the distance between the clustering centers [17]. The path of each particle of the swarm is influenced by the path of the best particle in the whole swarm, as all swarm particles are attracted quickly and simultaneously to the best particle in the search space. The optimal solution was reached according the following equations $[11,18]$ :

$$
f=\frac{\sum_{k=1}^{c n} \sum \forall D p \in C l_{k} d\left(D p, c_{-} c e n t_{k}\right) / D \sin k}{c n}
$$

Where $c$ represent number of cluster, $\mathrm{Cl}$ represent Cluster, $D p$ are Data pixel, $c_{-}$cent are cluster center, and $D s$ means number of data set in cluster k.

$$
d\left(D p, c_{-} \text {cent }_{j}\right)=\sqrt{\sum_{k=1}^{n d}\left(D p_{k}-c_{-} \text {cent }_{j k}\right)^{2}}
$$

To improve the performance of this algorithm, the membership function is used from one of the fuzzy logic algorithms here we used the membership 
function that present in reference [19]. As shown in the following section.

In this research, a new system was proposed to perform the pressing process of color images and to obtain the best results for compressed images in terms of their conformity with the original image in addition to obtaining the least storage space required for storing the compressed data. Where a process of shrinking the color image data was carried out using a clustering process that shrinks the data and reduces the duplicates in it, and in order to ensure that the data is not lost during shrinking, the best algorithms are used for this. Where swarm intelligence was used because of its importance in finding the best solution to all problems, and here the particle swarm algorithm was used to perform the clustering process, using the principle of the traditional clustering algorithm by finding the Euclidean distance between each pixel and clusters centers and including this calculation within the equation to calculate the fitness function of the particle swarm algorithm This resulted in excellent clustered data, and then this data was coded using the Huffman algorithm, thus obtaining high-quality compressed images.

\section{The new method: Fuzzy gustafson kessel particle swarm method FPSO}

In this algorithm, the PSO method that used here in this research are hybridizing with fuzzy logic to perform the clustering operation of color image data. By adding the membership function to the particle swarm algorithm equation which based on its work on the traditional clustering algorithm by finding the Euclidean distance between each light point in the image with the cluster centers that selected or determined at the start of work. Features of fuzzy logic were used to improve the work of swarm intelligence algorithms by using membership function values that help the pixel go to the class to which it belongs faster, in addition to using mahalanobis distance in finding the similarity value between pixels and the centers of clusters to ensure the data clustering correctly and quickly. The membership function has a primary advantage in improving the clustering process, because each pixel has its own class belonging value, which can be used to classify the pixel correctly. Here, the particle swarm algorithm was hybridized with the Gustafson kessel algorithm. The addition of this membership function to the particle swarm algorithm equation has improved its work in finding the best solutions represented by the best centers of clusters to which the color image data belongs according to the following equations:

$$
\begin{aligned}
& P G k_{f}= \\
& \underline{\sum_{k=1}^{c n} \sum \forall D p \in C l_{k} m \operatorname{ship} \mu \operatorname{dist}\left(D p, c_{-} \text {cent }_{k}\right) / D \operatorname{Din} k}
\end{aligned}
$$

Where mship $\mu$ was membership function of Gustafson kessel, $c_{-}$cent represent cluster center and dist are mahalanobis distance between data pixel of image and cluster center.

$$
\begin{gathered}
\operatorname{mship}_{i j}=\frac{1}{\sum_{k=1}^{K} \frac{\text { dist }_{i j} \overline{\text { dist }_{k j}}}{\text { fuzzifier-1 }^{2}}} \\
\operatorname{dist}_{i j}^{2}=\left(D p_{j}-c_{-} \text {cent }_{i}\right)^{T} A_{i}\left(D p_{j}-c_{-} \text {cent }_{i}\right)(7)
\end{gathered}
$$

fuzzifier are positive value, here the fitness function represented by the symbol $P G k \_f$ is calculated by the above equation to perform the clustering operation for all color image data. Then, after obtaining the clustering data, it is coded to obtain highly efficient compressed data without data loss.

\section{Grey wolf optimizer algorithm GWO}

Grey wolf algorithm is a swarm intelligent algorithm. This algorithm mimics the social leadership and hunting behavior of grey wolves in nature. Where society is divided into four sections, the first of which is alpha and symbolized by the symbol $\alpha$ and beta and symbolized by the symbol $\beta$ and the delta and symbolized by the symbol $\delta$ and omega and symbolized by the symbol $\omega$ [12]. Grey wolves live in groups and is called the Alpha Leader, who is responsible for making decisions regarding hunting, waking time, sleeping place, etc. These decisions are given to the rest of the group members and they recognize the Leader's decisions by bending the tail down $[20,21]$. The main stages of prey hunting by grey wolf are as follows: 1) Tracking, Chasing, Approaching from prey. 2) Pursuing, Encircling, Harassing until it stops moving. 3) Attack. The grey wolf algorithm works by randomly generating the initial population for a number of wolves and then defining the objective function for the problem to be found the best solution. The objective function is calculated for each search agent. After the end of all agents, the alpha wolf position will be taken as the best prey position, and then the location of each search agent will be updated according to the locations of the wolves and update all their information and values. Then calculating the objective function for all search agents and updating the values of $\overrightarrow{P_{\alpha}}, \overrightarrow{P_{\beta}}$ and $\overrightarrow{P_{\delta}}$. Finally an alpha wolf is the optimal solution. And the rest of the values $\vec{A}_{1,2,3}$ and $\vec{C}_{1,2,3}$ represent the vector coefficient and 
$\vec{P}_{\alpha, \beta, \delta}$ represents the prey position vector according to the wolf alpha, beta, delta and the value of $\vec{P}_{1,2,3}$ represents the updated position of the wolves Alpha, beta, and delta. The vector $\vec{P}$ represents the current grey wolf position and finally $\vec{P}(t+1)$ represents the new grey wolf position vector i.e the updated. As with the following equations: [22, 23]

$$
\begin{gathered}
\overrightarrow{D_{\alpha}}=\left|\overrightarrow{C_{1}} \cdot \overrightarrow{P_{\alpha}}-\vec{P}\right| \quad \overrightarrow{D_{\beta}}=\left|\overrightarrow{C_{2}} \cdot \overrightarrow{P_{\beta}}-\vec{P}\right| \\
\quad \overrightarrow{D_{\delta}}=\left|\overrightarrow{C_{3}} \cdot \overrightarrow{P_{\delta}}-\vec{P}\right| \\
\overrightarrow{P_{1}}=\overrightarrow{P_{\alpha}}-\overrightarrow{A_{1}} \cdot\left(\overrightarrow{D_{\alpha}}\right) \quad \overrightarrow{P_{2}}=\overrightarrow{P_{\beta}}-\overrightarrow{A_{2}} \cdot\left(\overrightarrow{D_{\beta}}\right) \\
\overrightarrow{P_{3}}=\overrightarrow{P_{\delta}}-\overrightarrow{A_{3}} \cdot\left(\overrightarrow{D_{\delta}}\right) \quad \vec{P}(t+1)=\frac{\overrightarrow{P_{1}}+\overrightarrow{P_{2}}+\overrightarrow{P_{3}}}{3}
\end{gathered}
$$

To perform a clustering operation for image data using the GWO algorithm, a fitness function is used that calculates the Euclidean distance between the image data values and the centers of the specified clustering from the start of the work according to the following equation [12]:

$$
f i t=\sum_{j=1}^{c n} \sum_{i=1}^{\mid \text {Cent }_{j} \mid} \sigma\left(\text { cent }_{j}, \text { data }_{i}\right)^{2}
$$

Where $\sigma$ are the Euclidean distance between the cluster center or centroid that indexed by cent $t_{j}$ and $i t h$ data pixel of the image as the following equation:

$$
\begin{aligned}
& \sigma\left(\text { cent }_{j}, \text { data }_{i}\right)= \\
& \sqrt{\sum_{w i=1}^{\operatorname{dim}}\left(\text { cent }_{j, w i}-\text { data }_{i, w i}\right)^{2}}
\end{aligned}
$$

Where data $_{i, w i}$ represent the width dimension of ith data pixel that belong to cluster center $c^{2} t_{j}$. To evaluate the quality of each wolf, the fitness function fit mentioned in the above equation is used here. And every wolf tries to reduce its fitness value.

In this research, the clustering process was used to reduce the image data to be compressed, as the grey wolf algorithm was used and linked to the Euclidean distance of the traditional clustering in calculating the fitness function for this method and obtaining the best clustering process for the data as this method was better than the particle swarm method.

\section{The novel method: Fuzzy gustafson kessel grey wolf optimizer FGWO}

In this algorithm, the grey wolf algorithm GWO, was hybridized to perform the clustering process of color image data with fuzzy logic by adding the membership function of Gustafson kessel algorithm that using mahalanobis distance $m \operatorname{ship} \mu_{i j}=$ $\frac{1}{\sum_{k=1}^{K} \frac{d i s t_{i j} \text { dist }_{i j}}{2}}$ (the same as that adding in PSO algorithm) to the fitness function of grey wolf, which is used in its work to calculate the distance between each pixel in the image with the optimal cluster centers of wolves, as shown in the following equation:

$$
\begin{array}{r}
f i t=\sum_{j=1}^{c n} \sum_{i=1}^{\mid \text {Cent }_{j} \mid} \sigma\left(\text { cent }_{j}, \text { data }_{i}\right)^{2} \times \\
\operatorname{mship}_{i j}
\end{array}
$$

It has also been added to equations of updating wolves position. The addition of this membership function led to reduce the time spent on clustering the data, speed up the work and ensure its accuracy and an improvement in the algorithm's work in finding the best solutions represented by the best cluster centers to which the color image data belongs. As each wolf has $\mathrm{k}$ cluster centers.

In this research, and during the implementation of all its methods, it is evident that the best method to perform the clustering process of data and then encode it and obtain compressed data is the fuzzy grey wolf method, which is evident from the results obtained when implementing all the methods on various colored images and different background.

\section{Huffman algorithm}

It is a lossless algorithm, the goal of this algorithm is to obtain the lowest number of bits to represent the image data to be compressed to reduce the storage space required to store the data in it[24]. The basis of the work of this algorithm is to calculate the probability of the presence of each pixel in the image by calculating its frequencies and then its probability and these probabilities are arranged in descending order and then collect the two lowest probabilities and so the process continues until the last two probabilities are reached and then coding them and obtaining a codeword for each pixel[25]. And pixels that a few repeated will have the longest length of the codeword, while the pixels that repeat a lot, it has the shortest codeword and this helps in reducing the number of bits that represent the image data to be compressed [26].

When using Huffman algorithm directly on the image data, the compressed data is obtained well, but not of high quality or with an optimal storage space, so the enhancement of the compression process was required to perform a preparation of the image data before encoding it with Huffman, and here in this 
research the clustering process of the data was performed before used coding, swarm intelligence methods are used in this, and in turn have been developed to become high performance. When applied to various images, high quality compressed data with excellent storage space is obtained. Where the clustering process of image data added an important feature of the compression process, where the data was shrunk and then encoded, thus it became easy and flexible in the coding process, thus resulting in a high-performance compression.

\section{Compression operation measurements}

To measure the accuracy and efficiency of the methods used by research, the following measurements of the color image are calculated [9]:

$$
\begin{array}{r}
R M S E=\sqrt{\frac{1}{M^{2}}} \sum_{i=1}^{M} \sum_{j=1}^{M}[\operatorname{origimage}(i, j)- \\
\operatorname{resimage}(i, j)]^{2}(13)
\end{array}
$$

origimage represent original image, resimage are result image, then calculate the value RMSE of full color image RMSE all as following:

$$
\begin{gathered}
R M S E_{\text {all }}= \\
\sqrt{\frac{1}{3}\left(R M S E_{\text {red }}^{2}+R M S E_{\text {green }}^{2}+R M S E_{\text {blue }}^{2}\right)} \\
\frac{P S N R_{\text {all }}=}{P S N R_{\text {red color }}+P S N R_{\text {greencolor }}+P S N R_{\text {blue color }}} \\
3 \\
\text { Where: } P S N R=10 \log _{10}\left[\frac{\max \text { gray }}{M S E}\right] d B \\
\text { MSE }=\frac{1}{N}(\text { origimage }- \text { resimage })^{2} \\
\text { Compression Ratio }=\frac{\text { size of original image file }}{\text { size of compressed file }}(16) \\
\text { bits } / \text { pixels }=\frac{\text { Bits number }}{\text { Pixels number }}
\end{gathered}
$$

\section{Results and discussion}

In this research, a new method was used in the image compressing process, where swarm intelligence algorithms were used to perform the clustering process of image data for which to be compressed, and then to obtain clustering data. This data, in turn, a lossless compression method is used to perform the compressing process for this reduced data, as the huffman method for coding is used, which depends on the calculation of the probability of the presence of each value in the image data after performing the clustering process on it using swarm intelligence methods. In this research, several methods were used to perform the clustering process of image data before encoding it in the huffman method because of the importance of the clustering in reducing data by collecting similar data in different clusters or groups. Clustered image is compensated for each value in the cluster center to which the point belongs, thus data was preserved without losing it. Then the encoding process is performed by calculating the probability of each value being found in the resulting clustered image, performing the steps of the huffman algorithm and obtaining a compressed file which are values represented by a series of values of 0 and 1 where compressed images have been obtained with high efficiency. The images were wanted to be compressed are prepared by using four intelligent methods in which the colors of the images were divided into a group of clusters, namely: Particle Swarm Optimization PSO, Fuzzy Gustafson kessel Particle Swarm Optimization FPSO, Grey Wolf Optimization GWO, and Fuzzy Gustafson kessel Grey Wolf Optimization FGWO. Thus, clustered images will be obtained that are encoded using the Huffman encoding algorithm without losing the data, thereby obtaining compressed images with efficiency and high quality represented by the lowest number of bits.

The four intelligent clustered methods were applied to many color images with different lighting intensity rates, as well as with multiple colors and different dimensions. The dataset (images) that used here are bmp uncompressed images that takes from the internet. Research methods were initially applied to the cat image with dimensions of $317 * 159$, where image data was compiled using a particle swarm optimization algorithm, an efficient method, and a clustered image was obtained on which the huffman compression algorithm was applied. Where a compressed image of appropriate quality was obtained but not at the required level, so a particle swarm optimization algorithm was developed to obtain a fuzzy particle swarm optimization algorithm that was also used to perform the clustering process on the image data that allows for easy and flexible fragmentation. When applied, its performance was better than the particle swarm optimization algorithm. After the fuzzy intelligent clustering, the clustered data was encoded with the huffman, where a better image was produced than the previous method. Grey wolf method was also used to perform the clustering process for the images to be compressed, where the clustering process in this way was better than the previous methods, and this algorithm was developed using fuzzy gustafson kessel to obtain a fuzzy intelligent clustering method used for clustering the data before it was encoded and its performance was 


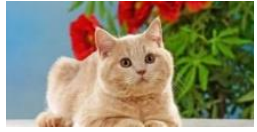

(a)

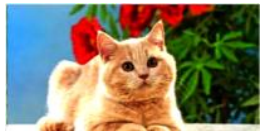

(d)

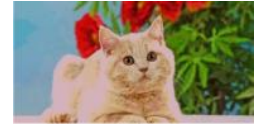

(b)

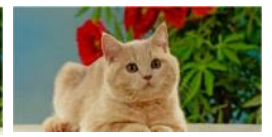

(e)

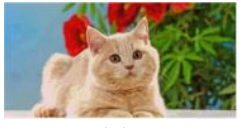

(c)
Figure. 2 Results of cat image with all methods: (a) original image, (b) reconstructed image by pso, (c) reconstructed image by fpso, (d) reconstructed image by gwo, and (e) reconstructed image by fgwo

Table 1. Measurements of all methods that used in research of a cat's image with dimensions $317 * 159$, number of light points 151209

\begin{tabular}{|l|l|l|l|l|}
\hline Measure & PSO & FPSO & GWO & FGWO \\
\hline MSE & 131.94 & 64.013 & 0.055 & 0.015 \\
\hline RMSE & 11.486 & 8.000 & 0.234 & 0.123 \\
\hline PSNR & 27.236 & 30.067 & 61.028 & 67.397 \\
\hline CR & $33.2: 1$ & $35.3: 1$ & $55.2: 1$ & $55.8: 1$ \\
\hline PNUM & 2217 & 2166 & 1593 & 1554 \\
\hline BITPP & 0.117 & 0.114 & 0.084 & 0.082 \\
\hline
\end{tabular}

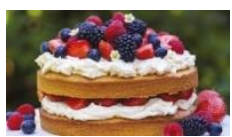

(a)

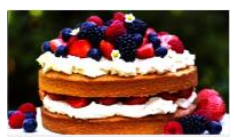

(d)

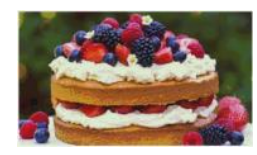

(b)

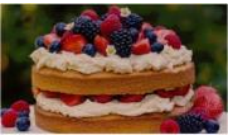

(e)
Figure. 3 Results of cake1 image with all methods: (a) original image, (b) reconstructed image by pso, (c) reconstructed image with fpso, (d) reconstructed image

by gwo, and (e) reconstructed image by fgwo

the best of all methods with a high compression ratio, the image quality is excellent and the size of the compressed file had the lowest size and the value of PSNR was the best value in this method for all the images that used in research. And the bit rate for each light point, this method has the lowest value among all the research methods.

And Figs. 2-16 shows the results of the compressing method that used in this research, where the first field of the figures represents the original image before pressing and the second field represents the reconstructed image from the compressed process using the PSO algorithm, and the third field of the table represents the result of the new method fuzzy intelligent method, and the fourth field represents the results of compressing for images using the grey wolf method, and the last field from the table represents the results of the last method of research represented by the novel method fuzzy grey wolf method, which was the best and efficient method.

To measure the performance of the compression methods used here, the MSE values, the RMSE values, and the PSNR values were calculated, as well as the compression ratio for each method and for all the color images used in the research, in addition to calculating the value of bits per pixels (BITPP), all of which are shown in Tables 1-15.

The following table demonstrates the results of the compressing process of color images dependent on the clustering data using swarm intelligence methods and hybridizing them with fuzzy logic, where, swarm intelligence techniques were used to obtain the best compression of color images, in addition to hybridization of these algorithms with fuzzy gustafson kessel to produce new and effective methods of compression, and when used in this research was give excellent results of all images that used here.

Table 2. Measurements of cake1 image with dimensions 299*168 and number of pixel 150696

\begin{tabular}{|l|l|l|l|l|}
\hline Measure & PSO & FPSO & GWO & FGWO \\
\hline MSE & 145.73 & 63.982 & 0.079 & 0.037 \\
\hline RMSE & 12.071 & 7.998 & 0.282 & 0.192 \\
\hline PSNR & 26.728 & 30.070 & 59.116 & 63.063 \\
\hline CR & $33.5: 1$ & $37.4: 1$ & $54.6: 1$ & $58.8: 1$ \\
\hline PNUM & 2196 & 1935 & 1596 & 1470 \\
\hline BITPP & 0.116 & 0.102 & 0.084 & 0.078 \\
\hline
\end{tabular}

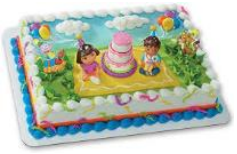

(a)

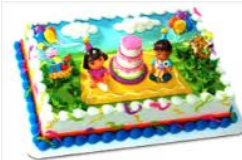

(d)

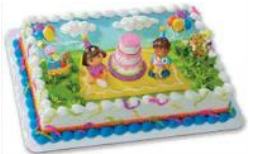

(b)

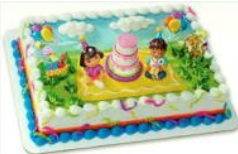

(e)
Figure. 4 Results of cake2 image with all methods: (a) original image, (b) reconstructed image with pso, (c) reconstructed image with fpso, (d) reconstructed image with gwo, and (e) reconstructed image with fgwo

Table 3. Results of cake 2 image with dimensions $279 * 180$ and number of pixel 150660

\begin{tabular}{|l|l|l|l|l|}
\hline Measure & PSO & FPSO & GWO & FGWO \\
\hline MSE & 116.70 & 63.980 & 0.239 & 0.061 \\
\hline RMSE & 10.802 & 7.998 & 0.489 & 0.248 \\
\hline PSNR & 27.962 & 30.070 & 54.334 & 60.495 \\
\hline CR & $37.5: 1$ & $46.8: 1$ & $56.5: 1$ & $62.03: 1$ \\
\hline PNUM & 1992 & 1569 & 1434 & 1344 \\
\hline BITPP & 0.105 & 0.083 & 0.076 & 0.071 \\
\hline
\end{tabular}




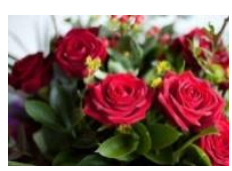

(a)

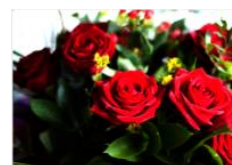

(d)

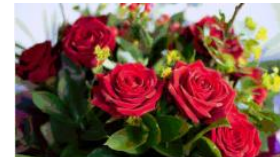

(b)

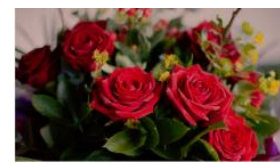

(e)

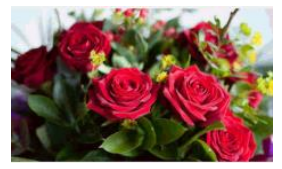

(c)
Figure. 5 Results of flower 1 image with all methods: (a) original image, (b) reconstructed image by pso, (c) reconstructed image by fpso, (d) reconstructed image by gwo, and (e) reconstructed image by fgwo

Table 4. Results of flower 1 image with dimensions $303 * 166$ and number of pixel 150894

\begin{tabular}{|l|l|l|l|l|}
\hline Measure & PSO & FPSO & GWO & FGWO \\
\hline MSE & 188.81 & 64.000 & 0.087 & 0.045 \\
\hline RMSE & 13.741 & 8.000 & 0.295 & 0.213 \\
\hline PSNR & 26.381 & 30.068 & 58.778 & 62.245 \\
\hline CR & $33.9: 1$ & $38.5: 1$ & $53.8: 1$ & $56.7: 1$ \\
\hline PNUM & 2163 & 1830 & 1602 & 1470 \\
\hline BITPP & 0.114 & 0.097 & 0.084 & 0.077 \\
\hline
\end{tabular}

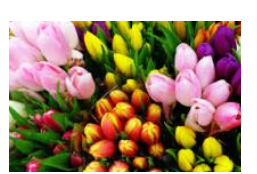

(a)

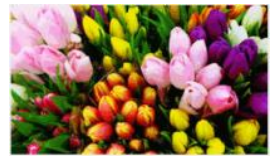

(b)

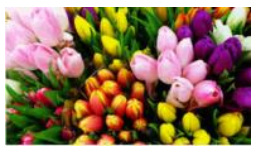

(c)

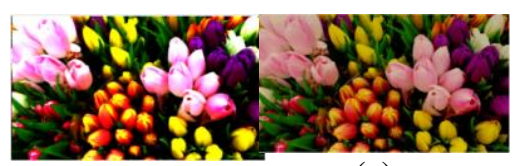

(d)

(e)

Figure. 6 Results of flower 2 image with all methods: (a) original image, (b) reconstructed image by pso, (c) reconstructed image by fpso, (d) reconstructed image by gwo, and (e) reconstructed image by fgwo

Table 5. Results of flower 2 image with Dimensions $300 * 168$ and 151200 pixels

\begin{tabular}{|l|l|l|l|l|}
\hline Measure & PSO & FPSO & GWO & FGWO \\
\hline MSE & 92.145 & 64.015 & 0.101 & 0.039 \\
\hline RMSE & 9.599 & 8.000 & 0.317 & 0.198 \\
\hline PSNR & 28.554 & 30.067 & 58.446 & 63.355 \\
\hline CR & $35.3: 1$ & $45.2: 1$ & $55.0: 1$ & $56.9: 1$ \\
\hline PNUM & 2061 & 1545 & 1518 & 1400 \\
\hline BITPP & 0.109 & 0.081 & 0.080 & 0.074 \\
\hline
\end{tabular}

From all the previous results, it is noted that the highest compression ratio was obtained through the Huffman method based on a new method of the grey wolf swarm hybridized with fuzzy gustafson kessel.

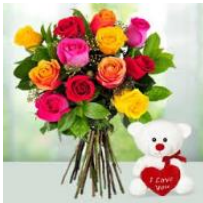

(a)

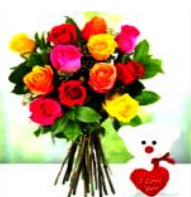

(d)

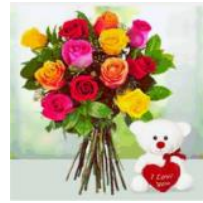

(b)

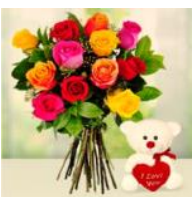

(e)

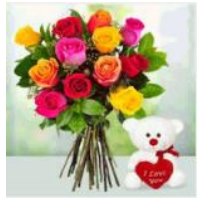

(c)
Figure. 7 Results of Flower3 image with all methods: (a) original image, (b) reconstructed image by pso, (c) reconstructed image by fpso, (d) reconstructed image by gwo, and (e) reconstructed image by fgwo

Table 6. Results of flower 3 image with dimensions $225 * 225$ and 151875 pixels

\begin{tabular}{|l|l|l|l|l|}
\hline Measure & PSO & FPSO & GWO & FGWO \\
\hline MSE & 131.70 & 63.994 & 0.185 & 0.035 \\
\hline RMSE & 11.476 & 7.999 & 0.430 & 0.189 \\
\hline PSNR & 27.351 & 30.069 & 55.516 & 64.671 \\
\hline CR & $35.3: 1$ & $54.2: 1$ & $54.8: 1$ & $56.5: 1$ \\
\hline PNUM & 2250 & 1398 & 1020 & 510 \\
\hline BITPP & 0.118 & 0.073 & 0.053 & 0.026 \\
\hline
\end{tabular}

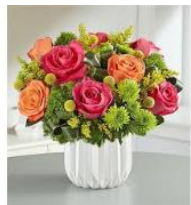

(a)

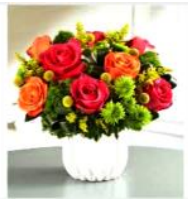

(d)

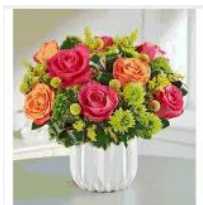

(b)

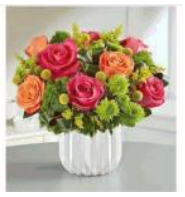

(c)

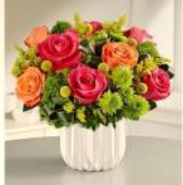

(e)
Figure. 8 Results of flower 4 image with all methods: (a) original image, (b) reconstructed image by pso, (c) reconstructed image by fpso, (d) reconstructed image by gwo, and (e) reconstructed image by fgwo

Table 7. Results of flower 4 image with dimensions $225 * 225$ and 151875 pixels

\begin{tabular}{|l|l|l|l|l|}
\hline Measure & PSO & FPSO & GWO & FGWO \\
\hline MSE & 153.97 & 63.985 & 0.1584 & 0.039 \\
\hline RMSE & 12.408 & 7.999 & 0.398 & 0.198 \\
\hline PSNR & 26.894 & 30.069 & 56.147 & 63.980 \\
\hline CR & $34.9: 1$ & $44.4: 1$ & $53.2: 1$ & $56.5: 1$ \\
\hline PNUM & 1468 & 1296 & 1036 & 512 \\
\hline BITPP & 0.077 & 0.068 & 0.054 & 0.026 \\
\hline
\end{tabular}




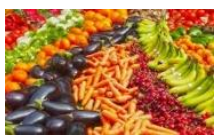

(a)

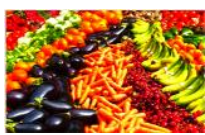

(d)

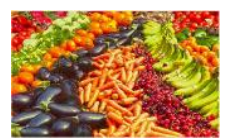

(b)

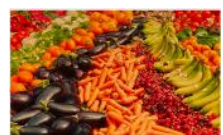

(e)

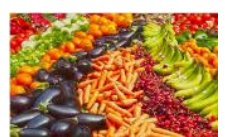

(c)
Figure. 9 Results of fruit 1 image with all methods: (a) original image, (b) reconstructed image by pso, (c) reconstructed image by fpso, (d) reconstructed image by gwo, and (e) reconstructed image by fgwo

Table 8. Results of fruit1 image with dimensions $290 * 174$ and 151380 pixel

\begin{tabular}{|l|l|l|l|l|}
\hline Measure & PSO & FPSO & GWO & FGWO \\
\hline MSE & 138.79 & 64.019 & 0.089 & 0.032 \\
\hline RMSE & 11.781 & 8.001 & 0.298 & 0.181 \\
\hline PSNR & 27.088 & 30.067 & 59.523 & 63.208 \\
\hline CR & $32.7: 1$ & $40.9: 1$ & $53.2: 1$ & $59.2: 1$ \\
\hline PNUM & 1510 & 1290 & 805 & 502 \\
\hline BITPP & 0.079 & 0.068 & 0.042 & 0.026 \\
\hline
\end{tabular}

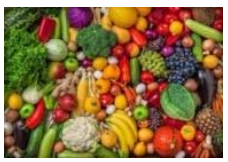

(a)

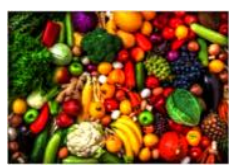

(d)

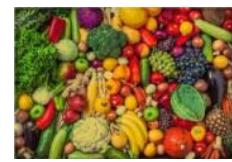

(b)

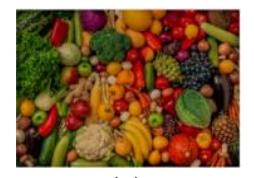

(e)

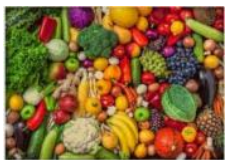

(c)
Figure. 10 Results of fruit 2 image with all methods: (a) original image, (b) reconstructed image by pso, (c) reconstructed image by fpso, (d) reconstructed image by gwo, and (e) reconstructed image by fgwo

Table 9. Results of fruit 2 image with Dimensions $270 * 186$ and 150660 pixel

\begin{tabular}{|l|l|l|l|l|}
\hline Measure & PSO & FPSO & GWO & FGWO \\
\hline MSE & 166.63 & 64.020 & 0.055 & 0.030 \\
\hline RMSE & 12.908 & 8.001 & 0.236 & 0.174 \\
\hline PSNR & 26.833 & 30.067 & 60.989 & 64.046 \\
\hline CR & $33.4: 1$ & $34.8: 1$ & $53.4: 1$ & $59.04: 1$ \\
\hline PNUM & 2136 & 2094 & 1605 & 1491 \\
\hline BITPP & 0.113 & 0.111 & 0.085 & 0.079 \\
\hline
\end{tabular}

The results were even better than the standard JPEG2000 compression method implemented on the standard $256 * 256$ peppers image in the reference [27] where a value of $0.24 \mathrm{bit} /$ pixel was obtained, the ratio of bits per light point, and when the JPEG algorithm was applied, a value of $1.57 \mathrm{bit} / \mathrm{pixel}$ was

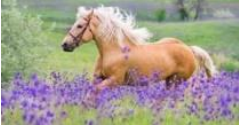

(a)

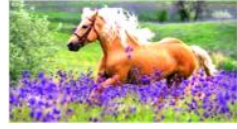

(d)

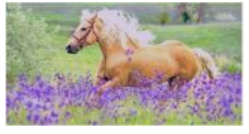

(b)

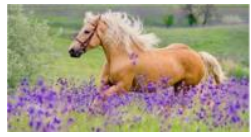

(e)

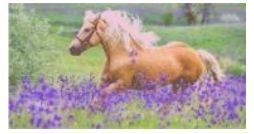

(c)
Figure. 11 Results of horse image with all methods: (a) original image, (b) reconstructed image by pso, (c) reconstructed image by fpso, (d) reconstructed image by gwo, and (e) reconstructed image by fgwo

Table 10. Results of horse image with dimensions $310 * 163$ and 151590 pixel

\begin{tabular}{|l|l|l|l|l|}
\hline Measure & PSO & FPSO & GWO & FGWO \\
\hline MSE & 84.352 & 63.978 & 0.046 & 0.008 \\
\hline RMSE & 9.184 & 7.998 & 0.216 & 0.090 \\
\hline PSNR & 29.284 & 30.070 & 61.488 & 70.052 \\
\hline CR & $34.8: 1$ & $35.0: 1$ & $53.4: 1$ & $57.8: 1$ \\
\hline PNUM & 2205 & 1492 & 1080 & 1000 \\
\hline BITPP & 0.116 & 0.078 & 0.056 & 0.052 \\
\hline
\end{tabular}

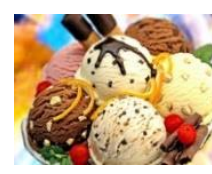

(a)

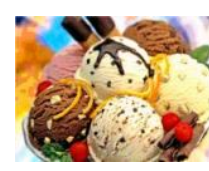

(b)

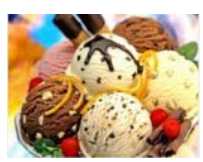

(c)

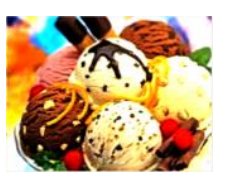

(d)

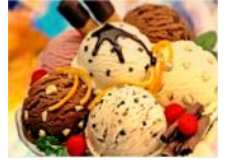

(e)
Figure. 12 Results of ice-cream image with all methods: (a) original image, (b) reconstructed image by pso, (c) reconstructed image by fpso, (d) reconstructed image by gwo, and (e) reconstructed image by fgwo

Table 11. Results of Ice-cream image with dimensions $259 * 194$ and 150738 pixel

\begin{tabular}{|l|l|l|l|l|}
\hline Measure & PSO & FPSO & GWO & FGWO \\
\hline MSE & 71.884 & 64.002 & 0.167 & 0.034 \\
\hline RMSE & 8.478 & 8.000 & 0.409 & 0.185 \\
\hline PSNR & 29.678 & 30.068 & 56.285 & 64.526 \\
\hline CR & $35.3: 1$ & $46.5: 1$ & $53.2: 1$ & $54.8: 1$ \\
\hline PNUM & 2235 & 1731 & 1647 & 1599 \\
\hline BITPP & 0.118 & 0.091 & 0.087 & 0.084 \\
\hline
\end{tabular}

obtained. While the methods of my research when carried out on the same image, a value of 0.03 bit/pixel was obtained, and from this it appears that the compression method based-clustering with the grey wolf swarm in my research was the best even when compared with standard compression methods, this is because in my research methods, 


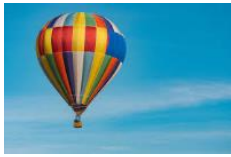

(a)

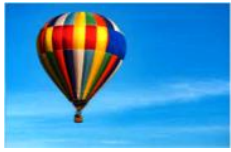

(d)

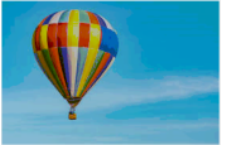

(b)

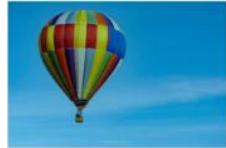

(e)

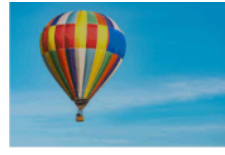

(c)

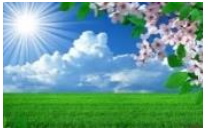

(a)

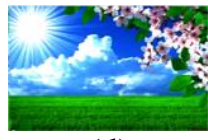

(d)

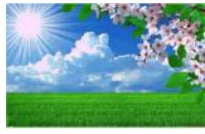

(b)

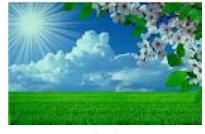

(e)

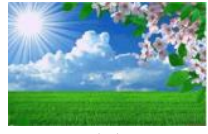

(c)
Figure. 15 Results of natural 1 image with all methods: (a) original image, (b) reconstructed image by pso, (c) reconstructed image by fpso, (d) reconstructed image by gwo, and (e) reconstructed image by fgwo

original image, (b) reconstructed image by pso, (c) reconstructed image by fpso, (d) reconstructed image by gwo, and (e) reconstructed image by fgwo

Table 12. Results of baloon 1 image with dimensions $275 * 183 \& 150975$ pixel

\begin{tabular}{|l|l|l|l|l|}
\hline Measure & PSO & FPSO & GWO & FGWO \\
\hline MSE & 143.93 & 64.053 & 0.041 & 0.031 \\
\hline RMSE & 11.997 & 8.003 & 0.202 & 0.176 \\
\hline PSNR & 28.109 & 30.065 & 62.700 & 65.008 \\
\hline CR & $35.8: 1$ & $37: 1$ & $55.6: 1$ & $58.04: 1$ \\
\hline PNUM & 1508 & 1474 & 1016 & 978 \\
\hline BITPP & 0.079 & 0.078 & 0.053 & 0.051 \\
\hline
\end{tabular}

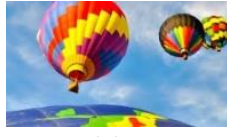

(a)

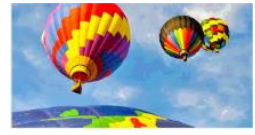

(b)

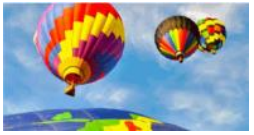

(c)

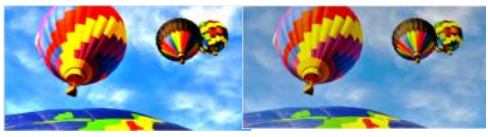

(d)

(e)

Figure 14: Results of baloon 2 image with all methods: (a) original image, (b) reconstructed image by pso, (c) reconstructed image by fpso, (d) reconstructed image by gwo, and (e) reconstructed image by fgwo

Table 13. Results of baloon 2 image with dimensions $316 * 159 \& 150732$ pixel

\begin{tabular}{|l|l|l|l|l|}
\hline Measure & PSO & FPSO & GWO & FGWO \\
\hline MSE & 63.785 & 63.994 & 0.066 & 0.008 \\
\hline RMSE & 7.986 & 7.999 & 0.310 & 0.092 \\
\hline PSNR & 30.545 & 30.069 & 58.563 & 69.680 \\
\hline CR & $33.3: 1$ & $34.9: 1$ & $54.8: 1$ & $55.06: 1$ \\
\hline PNUM & 2259 & 2010 & 1581 & 1502 \\
\hline BITPP & 0.119 & 0.106 & 0.083 & 0.080 \\
\hline
\end{tabular}

lossy compression methods were not used, but on the contrary, lossless compression methods were used by using the fuzzy clustering process with the intelligent swarm of image before encoding it in the Huffman method, because of the importance of the clustering
Table 14. Results of natural 1 image with dimensions $284 * 177 \& 150804$ pixel

\begin{tabular}{|l|l|l|l|l|}
\hline Measure & PSO & FPSO & GWO & FGWO \\
\hline MSE & 103.21 & 63.981 & 0.098 & 0.017 \\
\hline RMSE & 10.159 & 7.998 & 0.313 & 0.131 \\
\hline PSNR & 28.287 & 30.070 & 58.467 & 67.170 \\
\hline CR & $33.2: 1$ & $35.1: 1$ & $53.8: 1$ & $56.76: 1$ \\
\hline PNUM & 2322 & 2085 & 1593 & 1024 \\
\hline BITPP & 0.123 & 0.110 & 0.084 & 0.054 \\
\hline
\end{tabular}

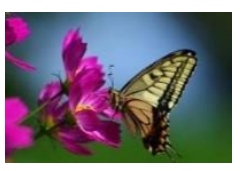

(a)

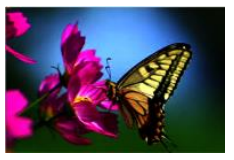

(d)

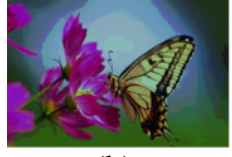

(b)

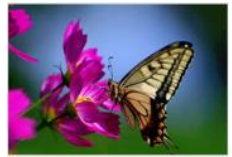

(e)
Figure. 16 Results of Butterfly image with all methods: (a) original image, (b) reconstructed image by pso, (c) reconstructed image by fpso, (d) reconstructed image by gwo, and (e) reconstructed image by fgwo

Table 15. Results of butterfly image with dimensions $275 * 183 \& 150975$ pixel

\begin{tabular}{|l|l|l|l|l|}
\hline Measure & PSO & FPSO & GWO & FGWO \\
\hline MSE & 295.54 & 64.002 & 0.049 & 0.080 \\
\hline RMSE & 17.191 & 8.000 & 0.222 & 0.282 \\
\hline PSNR & 25.508 & 30.068 & 61.210 & 60.077 \\
\hline CR & $32.3: 1$ & $35.3: 1$ & $54.0: 1$ & $59.2: 1$ \\
\hline PNUM & 2325 & 2220 & 1608 & 1500 \\
\hline BITPP & 0.123 & 0.117 & 0.085 & 0.079 \\
\hline
\end{tabular}

in collecting similar data into different groups or clusters, and compensating for each value of image by the center of the cluster that the point belongs to it, as this resulted lossless data. Also in [9] the values of the PSNR measure ranged between 32.6901 39.0999, while in our research and when applying the 
proposed method FGWO, the values of the PSNR measure ranged between 60.496-70.052 and thus quality of new methods was proven. Finally in [10] the highest PSNR values were obtained, which were 56.804, and the compression ratio was 44.342. Whereas in our research, better values of these measurements were obtained where the values of the PSNR measure ranged from 60.496-70.052 and the values of the compression ratio ranged between 54.85-62.03, and thus the research methods proved their quality.

\section{Conclusion}

Digital Image Compression is considered one of the most important technologies used in the current world as a result of the development of modern technology. So the trend increased to find efficient compression methods. In this research, a new method was proposed to compress color images based on the image data clustering using swarm intelligence algorithms. To improve the pressing process, these algorithms were developed using fuzzy logic to make the clustering process easy and flexible. Then, huffman algorithm was applied to the clustered data to obtain a highly efficient compressed file with little storage space. It was noted that the process of pressing with the algorithm of compressing without loss gave a high percentage of compressing, in addition to the resulting image was similar to the original image and with high quality. Four methods of compressing were applied in this research to various color images of lighting and dimensions. The results showed that the first method PSO gave a good result, but in some images the reconstructed image have a kind of distortion, so the second developed method FPSO was used to perform the compress and gave better results than the first. Then another swarm algorithm was used for the clustering and then compressed the clustered data, as this method GWO gave excellent results and when developed as its predecessor by hybridizing it with fuzzy Then another swarm algorithm was used for the clustering and then compressed the clustered data, as this method GWO gave excellent results and when developed as its predecessor by hybridizing it with fuzzy logic FGWO, FGWO, the best results were obtained from all aspects, whether by looking or through all the calculated mathematical measurements MSE,RMSE,PSNR,CR,POINT NUM AND BITSPERPIXEL shown in the previous tables. From the previous results, it is evident that the cluster-based compression method using swarm intelligence as well as hybridization with the fuzzy Gustafson kessel algorithm has proven its success and accuracy by obtaining satisfactory results for the pressing process.

\section{Conflicts of Interest}

Declare conflicts of interest or state "The author declares no conflict of interest." Authors must identify and declare any personal circumstances or interest that may be perceived as inappropriately influencing the representation or interpretation of reported research results.

\section{Author Contributions}

For research articles with several authors, a short paragraph specifying their individual contributions must be provided. The following statements should be used as follows: "Conceptualization, Shahbaa; methodology, Shahbaa; software, Shahbaa; validation, Shahbaa; formal analysis, Shahbaa; investigation, Shahbaa; resources, Shahbaa; data curation, Shahbaa; writing - original draft preparation, Shahbaa; writing - review and editing, Shahbaa; visualization, Shahbaa; supervision, Shahbaa; project administration, Shahbaa; funding acquisition, Shahbaa", etc. Authorship must be limited to those who have contributed substantially to the work reported.

\section{Acknowledgments}

The author would like to thank the University of Mosul in Iraq for providing Moral support during the completion of this research.

\section{References}

[1] J. As-ad, M. Rahaman, R. Mustafa, Z. Sultana, and L. Nahar, "An Improved Decoding Technique for Efficient Huffman Coding", Journal of Computer Science and Applications and Information Technology, Vol. 2, No. 1, pp. $1-5,2017$.

[2] B. Aruna, D. Srinivas, and R. Boda, "Fast Huffman Coding Scheme Implementation On FPGA", International Journal of Advance Engineering and Research Development, Vol. 4, No. 12, pp. 478-483, 2017.

[3] C. Singh, A. Gupta, "Huffman Algorithm Improvement", International Journal of Computer Science and Information Technology Research. Vol. 5, No. 1, pp. 22-24, 2017.

[4] M. Tokovarov, "Modification of Adaptive Huffman Coding for use in encoding large alphabets", ITM Web of Conf. 15, pp. 1-6, 2017.

[5] M. Mathur, S. Loonker, and D. Saxena, "Lossless Huffman Coding Technique for Image Compression and Reconstruction using Binary 
Trees", Int.J.Comp. Tech.Appl, Vol. 3, No. 1, pp. 76-79, 2012.

[6] M. Abd Rahman, B. Ismail, K. Naidu, and M. Rahmat, "Review on Population-Based Metaheuristic Search Techniques for Optimal Power Flow", Indonesian Journal of Electrical Engineering and Computer Science, Vol. 15, No. 1, pp. 373-381, 2019.

[7] P. Chalotra and H. Kaur, "Optimizing Fuzzy Clustering using Swarm Intelligence in Data Mining", International Journal of Advanced Research in Computer Science, Vol. 2, No. 4, pp. 34-37, 2011.

[8] B. Khaleel, "Image Clustering based on Artificial Intelligence Techniques", AL-Rafidain Journal of Computer Sciences and Mathematics, Vol. 11, No. 1, pp. 99-112, 2014.

[9] P. Kaur and E. Singh, "Integrated Particle Swarm Optimization and Genetic Algorithm Based Compression for Reduction of Blocking Artifacts", International Journal of Computer Science and Information Technologies (IJCSIT), Vol. 6, No. 5, pp. 4520-4527, 2015.

[10] S. keerthika and S. Vidhya, "Fractal Image Compression with Advanced Particle Swarm Optimization and Sorting", International Journal of Computer Science Trends and Technology (IJCST), Vol. 4, No. 5, pp. 309-312, 2016.

[11] H. Li, S. Chen, and D. Zhao, "A Clustering Method for Electromagnetic Interference Signals Based on Particle Swarm Optimization", MATEC Web of Conf. 61, 2016.

[12] I. Aljarah, M. Mafarja, A. Heidari, H. Faris, and S. Mirjalili, "Clustering analysis using a novel locality-informed grey wolf-inspired clustering approach", Knowledge and Information Systems, Vol. 62, pp. 507-539, 2020.

[13] D. Divya and S. Prakasha, "Clustering Techniques for Medical Imaging", International Journal of Innovative Technology and Exploring Engineering (IJITEE), Vol. 9, No. 2S, pp. 510516, 2019.

[14] B. Khaleel, "Using Artificial Intelligence Techniques for Image Compression", $A L$ Rafidain Journal of Computer Sciences and Mathematics, Vol. 11, No. 2, pp. 65-81, 2014.

[15] S. Khaleel and R. khaled, "Selection and Prioritization of Test Cases by using Bees Colony", AL-Rafidain Journal of Computer Sciences and Mathematics, Vol. 11, No. 1, pp. 179-201, 2014.

[16] S. Khaleel and A. Al Thanoon, "Design a Tool for Generating Test Cases using Swarm Intelligence", AL-Rafidain Journal of Computer
Sciences and Mathematics, Vol. 10, No. 1, pp. 421-444, 2013.

[17] S. Khaleel, K. Saleh, "Detection of Network Anomaly Based on hybrid intelligence techniques", AL-Rafidain Journal of Computer Sciences and Mathematics, Vol. 9, No. 2, pp. 8198, 2012.

[18] S. Akojwar and P. Kshirsagar, "A Novel Probabilistic-PSO Based Learning Algorithm for Optimization of Neural Networks for Benchmark Problems", Wseas Transactions on Electronics, Vol. 7, pp. 79-84, 2016.

[19] B. AL-Taey, "Artificial Intelligent Techniques for Intrusion Detection and Classification", Ph.D. Thesis, Computer Science, College of Computer Science and Mathematics, University of Mosul, 2012.

[20] A. Jha, S. Jain, S. Thenmalar, "Survey On Grey Wolf Algorithm in Resource Allocation", International Journal of Pure and Applied Mathematics, Vol. 118, No. 22, pp. 201-206, 2018.

[21] H. Faris, I. Aljarah, M. Al-Betar, S. Mirjalili, "Grey wolf optimizer: a review of recent variants and applications", Neural Computing and Applications, Vol. 30, pp. 413-435, 2018.

[22] N. Singh and S. Singh, "A Modified Mean Gray Wolf Optimization Approach for Benchmark and Biomedical Problems", Evolutionary Bioinformatics, Vol. 13, pp. 1-28, 2017.

[23] N. Hatta, A. Mohd Zain, R. Sallehuddin, Z. Shayfull, and Y. Yusoff, "Recent studies on optimization method of Grey Wolf Optimizer (GWO): a review (2014-2017)", Artificial Intelligence Review, Vol. 52, pp. 2651-2683, 2019.

[24] M. Sharma, "Compression Using Huffman Coding", International Journal of Computer Science and Network Security IJCSNS, Vol. 10, No. 5, pp. 133-141, 2010.

[25] M. Singh, S. Kumar, S. Singh, Manish, "Various Image Compression Techniques: Lossy and Lossless", International Journal of Computer Applications, Vol. 142, No. 6, pp. 23-26, 2016.

[26] K. Satone, "Huffman Encoding Technique in Image Compression", International Conf. on Recent Trends in Engineering Science and Technology (ICRTEST 2017), (Special Issue 2122 January 2017), Vol. 5, No. 1, pp. 252-255, 2017.

[27] T. Acharya and A. Ray, Image Processing Principles and Applications, Wiley \& Sons Inc., Hoboken, New Jersey, 2005. 